\title{
COMPORTAMENTO DE EIXO VAZADO DURANTE O FORJAMENTO EM MATRIZ ABERTA ATRAVÉS DA SIMULAÇÃO NUMÉRICA E ATRAVÉS DE EXPERIMENTO FÍSICO
}

Fábio Junkes Corrêa ${ }^{1,2}$ Lírio Schaeffer ${ }^{3}$

\section{Resumo}

Forjamento em matriz aberta é um processo de forjamento largamente empregado para produção de eixos de grande porte como, por exemplo, eixo de transmissão para os aero geradores. Visando a redução de peso, busca-se produzir um eixo vazado que atenda as mesmas condições de trabalho de um eixo maciço. Durante o forjamento de eixos de grande porte, o eixo sofre compressões sucessivas gerando um campo de tensões que, através da simulação numérica, evidenciam-se as diferenças na distribuição de tensões ao longo da seção. Analisou-se o forjamento em matriz aberta de um tarugo cilíndrico vazado com o intuito de produzir um eixo vazado através de simulações computacionais pelo Método dos Elementos Finitos e, com base de experimentos físicos, constataram-se a validade da simulação numérica. Realizaram-se simulações baseado no FEM, utilizando o software Simufact.Forming 12.0, obtendo-se valores de distribuição de tensões e deformações. Observou-se que as regiões que apresentaram maiores concentrações de deformações equivalentes coincidiram com as regiões que apresentaram maiores concentrações de tensões na direção $z$ encontradas nos resultados da simulação numérica, seguindo a tendência esperada. Através de experimentos físicos foi constatado que os valores das forças para cada recalque apresentado pelo software de simulação numérica ficaram bem próximos da realidade, validando a empregabilidade do uso da simulação numérica.

Palavras-chave: Forjamento em matriz aberta; Método dos elementos finitos; Experimento físico.

\section{HOLLOW SHAFT BEHAVIOR DURING THE FORGING IN OPEN DIE THROUGH NUMERICAL SIMULATION AND THROUGH PHYSICAL EXPERIMENT}

\begin{abstract}
Open die forging is a forging process widely used for the production of large size axes as, for example, drive shaft for wind turbine. In order to reduce weight, it seeks to produce a hollow shaft that meets the same working conditions of a solid shaft. During the forging of large size shaft, the part undergoes successive compressions generating a field stress that show the differences in stress distribution throughout section through numerical simulation. It analyzed the open die forging of a hollow cylindrical billet in order to produce a hollow shaft through computer simulation by the finite element method and by physical experiments verified the validity of the numerical simulation. Simulations were performed based on FEM, using the software Simufact.Forming 12.0, getting the stress and strain distribution values. It observed that the regions that showed greater concentrations equivalent strain coincided with the regions that showed greater stress concentrations in the $z$ direction found in the numerical simulation results following the expected trend. Through physical experiments it was verified that the values of forces for each upset presented by numerical simulation software were very close to reality, validating the employability of the use of numerical simulation.
\end{abstract}

Keywords: Open die forging; Finite element method; Physical experiment.

'Universidade Tecnológica Federal do Paraná - UTFPR, Francisco Beltrão - PR, Brasil. E-mail: fabio.correa@ufrgs.br

${ }^{2}$ Laboratório de Transformação Mecânica - LdTM, Departamento de Metalurgia, Universidade Federal do Rio Grande do Sul - UFRGS, Porto Alegre, RS, Brasil.

${ }^{3}$ Programa de Pós-graduação em Engenharia de Minas, Metalúrgica e de Materiais - PPGEM, Laboratório de Transformação Mecânica - LdTM, Departamento de Metalurgia, Universidade Federal do Rio Grande do Sul - UFRGS, Porto Alegre, RS, Brasil.

2176-1523 (C) 2017 Associação Brasileira de Metalurgia, Materiais e Mineração. Publicado pela ABM. Este é um artigo de acesso aberto distribuído sob os termos da licença Creative Commons CC BY-NC'-ND (Attribution-NonCommercial-NoDerivs) - https:// creativecommons.org/licenses/by-nc-nd/4.0\%. 


\section{INTRODUÇÃO}

O forjamento, dependendo como escoar o material, pode ser classificado como forjamento em matriz fechada ou forjamento em matriz aberta. No forjamento em matriz aberta (Figura I), o material é forjado de forma livre, sem ou com pouca restrição conferida pelas matrizes, escoando nas direções laterais. Entretanto, o metal pode ser parcialmente confinado pelas matrizes $[1,2]$.

No forjamento em matriz aberta (Figura I), a peça é conformada com matrizes de formato simples, quando comparadas com o forjamento em matriz fechada aonde são usadas matrizes com geometrias mais complexas [3].

Os tamanhos das ferramentas são pequenas comparadas com o tamanho total das peças forjadas. Como a peça a ser forjada costuma ser maior do que as ferramentas, a deformação costuma ser restrita a uma pequena região da peça e, com isso, as cargas de forjamento necessárias são menores do que aquelas necessárias para deformar a peça inteira. Portanto, uma máquina de menor capacidade pode ser usada para produzir uma determinada peça, empregando forças de forjamento reduzidas progressivamente [4].

Componentes mecânicos de grande porte como ganchos, correntes, âncoras, alavancas, mancais, eixos de turbinas encontrados em aviões, navios e de aero geradores, rolos de laminação, e outros componentes usados na indústria mecânica pesada são submetidos ao processo de forjamento em matriz aberta. Esse processo de fabricação, subsequente a outros processos, é capaz de produzir peças com uma elevada relação resistência-peso e resistência ao impacto $[5,6]$.

No forjamento, a utilização do Método dos Elementos Finitos é uma alternativa conveniente aos métodos analíticos para a aquisição de informações detalhadas na análise do processo de forjamento, como a distribuição de deformações e tensões [7].

A simulação numérica tem aparecido nas indústrias com o propósito de substituir os métodos de convencionais, reduzindo os custos, tornando-se um método aceitável como ferramenta de trabalho. Dependendo dos resultados desejados, a escolha do programa adequado, dentre os disponíveis no mercado, é determinada de acordo com as necessidades específicas requisitadas. Normalmente é usado o Método dos Elementos Finitos [8].

O Método dos Elementos Finitos através da simulação numérica pode ser empregado para analisar a distribuição de deformações e a distribuição de tensões em uma operação de forjamento em matriz aberta. Os métodos numéricos mostram com bastante exatidão valores de deformações equivalentes e tensões nas direções principais em regiões específicas [9].

Neste trabalho, um tarugo cilíndrico vazado é submetido a um processo de forjamento em matriz aberta através da simulação numérica com uso do software Simufact. Forming 12.0, analisando-se a distribuição de deformações e a distribuição de tensões, constatando-os com a realização de experimento físico.

\section{MATERIAIS E MÉTODOS}

Para o forjamento em matriz aberta, foi selecionado um tarugo cilíndrico de $20 \mathrm{~kg}$ vazado com o material AISI 4I 40, cujo método está representado na Figura 2.

$\mathrm{Na}$ realização do forjamento em matriz aberta foi usado um par de matrizes planas, sendo uma superior e outra inferior (Figura 2). Para manter o furo interno foi introduzido um mandril com uma leve conicidade para facilitar a retirada do mandril após o forjamento. Essas ferramentas foram fabricadas com aço ferramenta $\mathrm{HI} 3$ para utilização nos experimentos físicos. Para utilização na simulação numérica, as ferramentas foram modeladas através do software Solidworks 2012.

Os equipamentos usados para a realização dos experimentos foram o forno resistivo convencional Linn Elektro Therm (Figura 3a), Modelo KK 55, com potência de $3,3 \mathrm{~kW}$, o mecanismo com um moto-redutor instalado
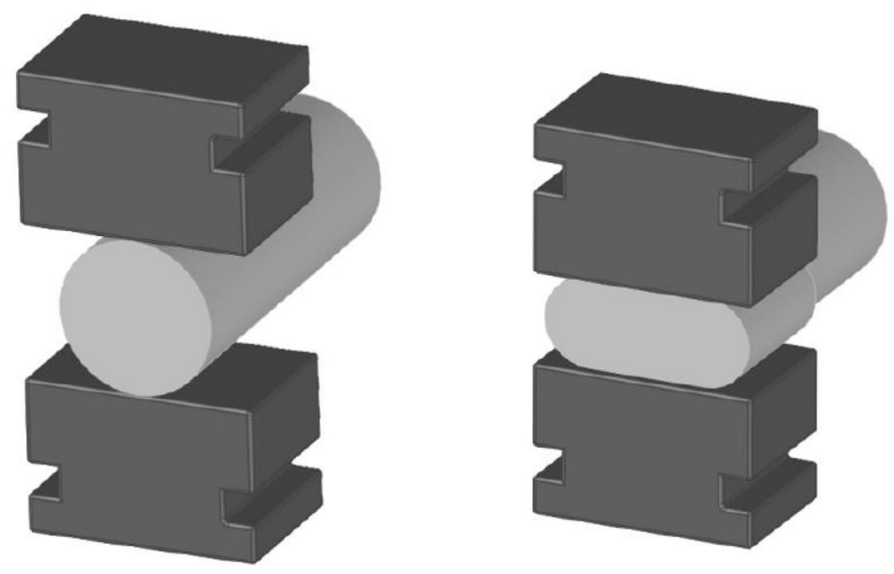

Figura I. Forjamento em matriz aberta. 
(Figura 3b), a prensa hidráulica da marca FKL (Figura 3c), modelo PH 600 , com capacidade de $6.000 \mathrm{kN}$ e a célula de carga (Figura 3d), com a capacidade de $3.000 \mathrm{kN}$. O forno resistivo foi utilizado para o aquecimento da peça, a prensa hidráulica para a aplicação dos recalques com uma velocidade de prensagem de $5 \mathrm{~mm} / \mathrm{s}$, a célula de carga para aferir os valores de força e o mecanismo para retirar a peça incandescente do forno e conduzir até o par de matrizes na prensa.

Para a realização da simulação numérica do processo de forjamento em matriz aberta, utilizou-se o software Simufact.Forming 12.0. Os dados de entrada para a simulação numérica são mostrados na Tabela $I$ os quais foram inseridos nas simulações. Os parâmetros térmicos, como o coeficiente de transferência de calor por convecção e por irradiação foram determinados conforme estão indicados no programa e, a emissividade foi alterada para 0,9 devida à superfície porosa presente na peça de trabalho durante 0 forjamento a quente, levando em consideração a formação de óxidos [10]. O coeficiente de atrito estabelecido entre a peça de trabalho e as ferramentas para a realização da simulação foi de $\mu=0,4$ [I I ]. Esse valor é substancialmente elevado em função de ser um processo de forjamento

Tabela I. Dados de entrada

\begin{tabular}{|c|c|c|}
\hline Temperatura da peça & 1.100 & ${ }^{\circ} \mathbf{C}$ \\
\hline Temperatura das Ferramentas & 30 & ${ }^{\circ} \mathrm{C}$ \\
\hline $\begin{array}{l}\text { Coeficiente de transferência de calor por } \\
\text { convecção }\end{array}$ & 50 & $\mathrm{~W} / \mathrm{m}^{2} \cdot \mathrm{K}$ \\
\hline $\begin{array}{l}\text { Coeficiente de transferência de calor por } \\
\text { irradiação }\end{array}$ & 20.000 & $\mathrm{~W} / \mathrm{m}^{2} . \mathrm{K}$ \\
\hline $\begin{array}{l}\text { Emissividade por radiação térmica ao meio } \\
\text { ambiente }\end{array}$ & 0,9 & - \\
\hline Atrito interfacial entre as ferramentas e a peça & 0,4 & - \\
\hline Material das Ferramentas & $\mathrm{H}-\mathrm{I} 3$ & - \\
\hline Material da Peça & SAE $4 \mid 40$ & - \\
\hline
\end{tabular}

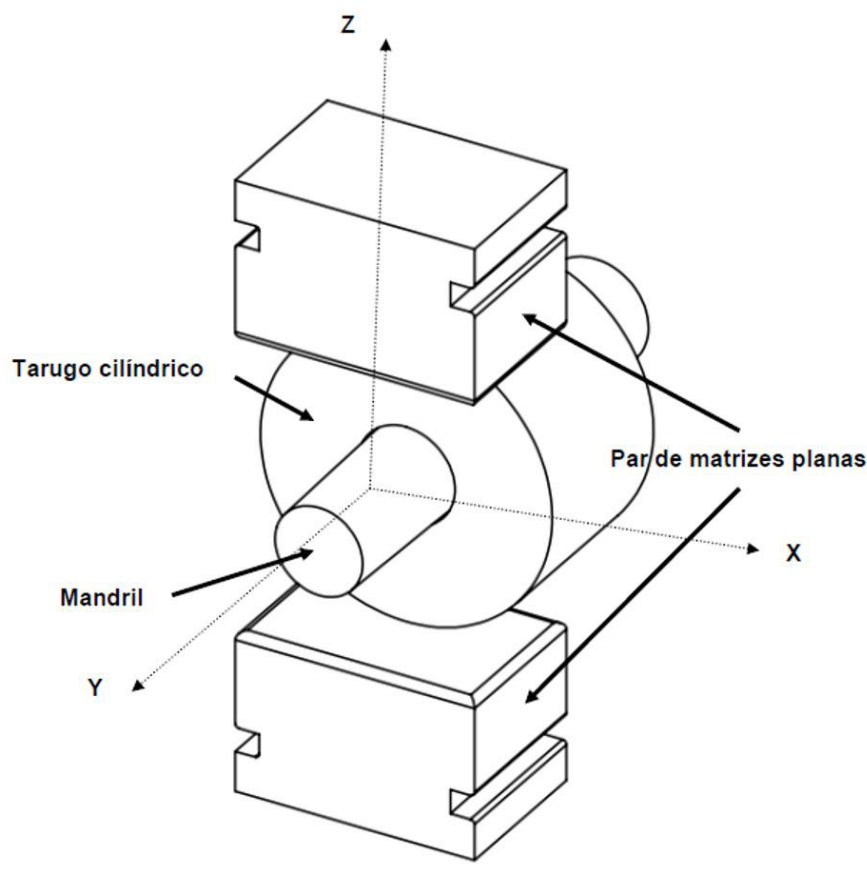

Figura 2. Montagem do forjamento do tarugo cilíndrico.
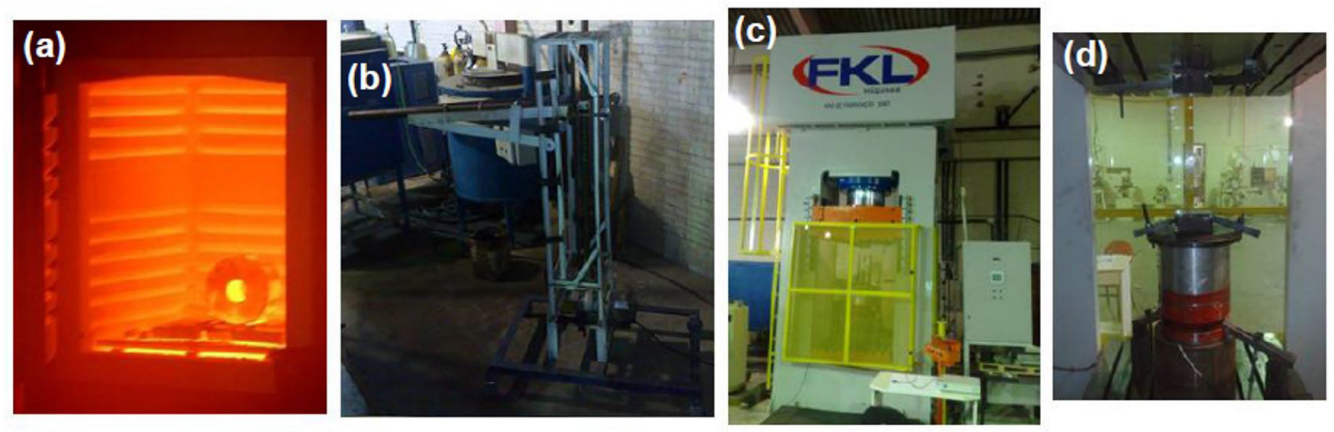

Figura 3. (a) Forno resistivo; (b) Mecanismo; (c) Prensa hidráulica; (d) Célula de carga. 
a quente, considerando que $\circ$ aumento da temperatura provoca o acréscimo do coeficiente de atrito, sabendo que a variação da temperatura altera as características da peça de trabalho. $O$ material definido para $\circ$ tarugo cilíndrico foi o aço AISI 4 I 40 e o material das matrizes foi o aço ferramenta $\mathrm{HI}$ 3. A temperatura estabelecida para peça foi de $1.100^{\circ} \mathrm{C}$ e, para as ferramentas, uma temperatura de $30^{\circ} \mathrm{C}$ (temperatura ambiente).

Sabendo que o forjamento em matriz aberta do tarugo cilíndrico vazado de $20 \mathrm{~kg}$ tem por objetivo seu alongamento para a produção de um eixo vazado, aplicaram-se dois recalques no tarugo cilíndrico através da simulação numérica. Primeiro foram posicionadas as ferramentas tangentes ao tarugo cilíndrico e aplicado o recalque reduzindo $10 \%$ do seu diâmetro. Após a aplicação do primeiro recalque, o tarugo foi rotacionado $90^{\circ} \mathrm{em}$ torno do seu eixo longitudinal (Figura 4) e aplicado outro recalque reduzindo 10\% do seu diâmetro.

Para a realização do experimento físico, inicialmente foi aquecido o tarugo cilíndrico a uma temperatura de $1.200^{\circ} \mathrm{C}$, e permanecido no forno durante o tempo necessário para estabelecer o pleno aquecimento da peça. Após o tempo de aquecimento, a peça foi retirada do forno com o uso do mecanismo, levado até as matrizes planas na prensa hidráulica e aplicado o primeiro recalque (Figura 4a) com uma redução de $10 \%$ em relação ao diâmetro inicial, reproduzindo a simulação numérica de forma experimental. Depois a aplicação do primeiro recalque, o tarugo foi conduzido ao forno resistivo para aquecer novamente até a temperatura de forjamento a quente. Após o tempo de aquecimento, o tarugo cilíndrico foi retirado do forno, conduzido até as matrizes, rotacionado $90^{\circ} \mathrm{em}$ torno de seu eixo e aplicado outro recalque (Figura $4 b$ ), repetindo o mesmo procedimento da simulação numérica.

\section{RESULTADOS}

Com a efetivação da simulação, obtiveram-se os valores de deformações e de tensões resultantes. Nas Figuras 5 e 6 são apresentados a distribuição de deformações equivalentes e de tensões na direção normal, respectivamente, resultantes da simulação numérica pelo Método dos Elementos Finitos.

Os resultados da simulação numérica possibilitam identificar heterogeneidades tanto na distribuição de deformações equivalente, como na distribuição de tensões normais na direção $z$, através da diferenciação das tonalidades das cores de cada elemento. Os valores máximos das deformações equivalentes foram encontrados nas regiões centrais do corpo forjado, evidenciando-se maior concentração nas regiões internas próximas do furo e menores concentrações nas regiões periféricas.

Em relação às tensões, além das regiões onde há o contato interfacial entre as matrizes e a peça de trabalho, também foram identificadas maiores concentrações de tensões normais na direção $z$ nas regiões centralizadas $e$ menores concentrações de tensões normais na direção $z$ nas regiões periféricas, justificando que maiores valores de deformações equivalentes causam aumento das tensões normais.

Além das deformações e tensões resultantes, o software também forneceu o comportamento da força requerida durante o forjamento, obtendo-se um valor máximo no final da simulação (Figura 7).

Para validar a simulação numérica, executaram-se experimentos físicos, obtendo-se os valores de força em função do deslocamento durante a aplicação de cada recalque. Nos experimentos físicos, os valores de forças foram extraídos do software Catman 3.2 através do sistema de aquisição de dados HBM que estava conectado à célula de carga a qual detectava valores de forças instantâneas durante o forjamento. Na Figura 8 são apresentados os gráficos das forças em função do deslocamento para ambos os recalques destacando o valor máximo no final do experimento.

(a)
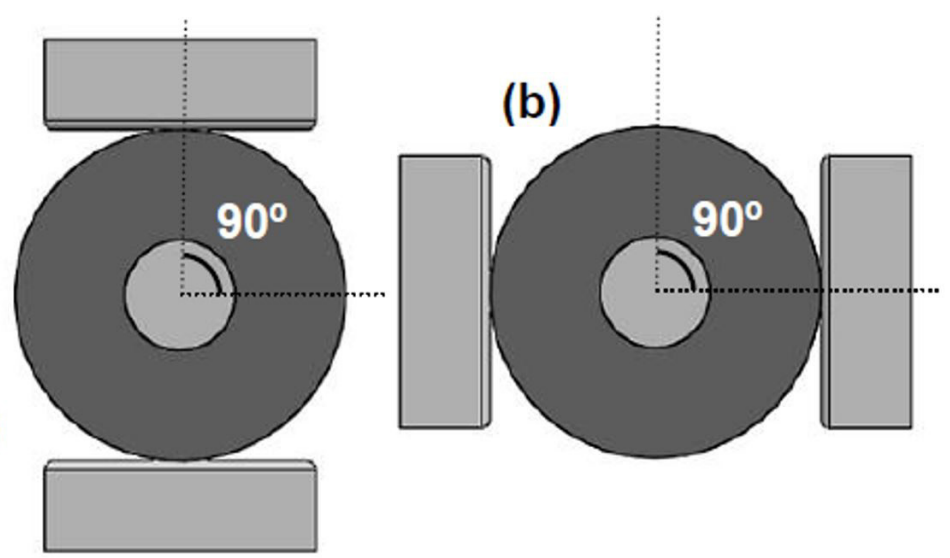

Figura 4. Aplicação do (a) primeiro recalque; (b) segundo recalque. 


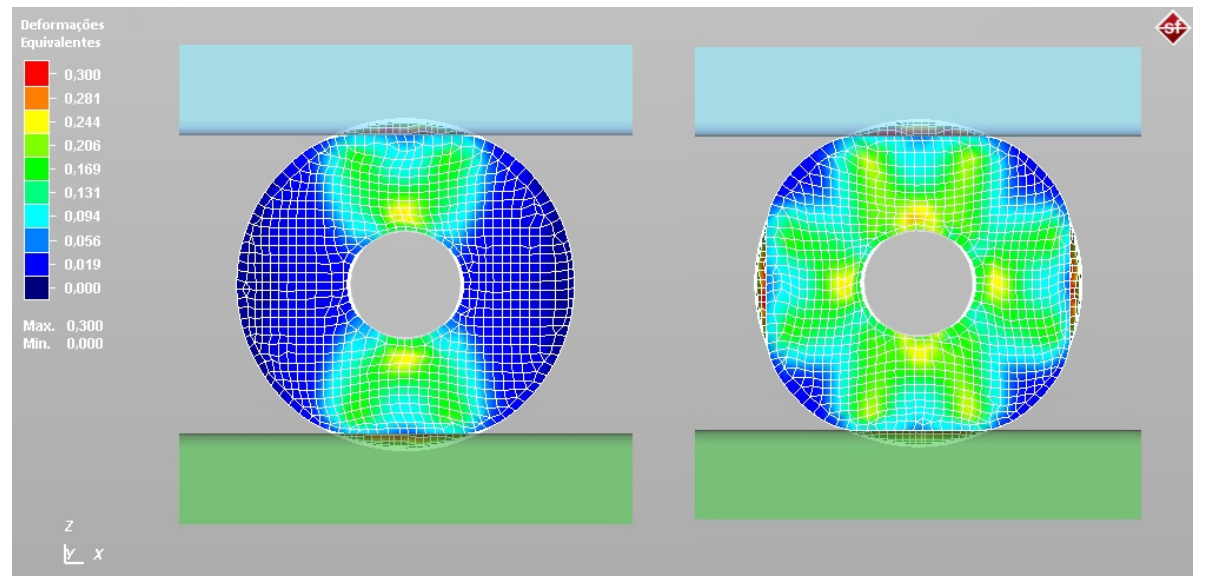

Figura 5. Distribuição das deformações equivalentes para o primeiro recalque.

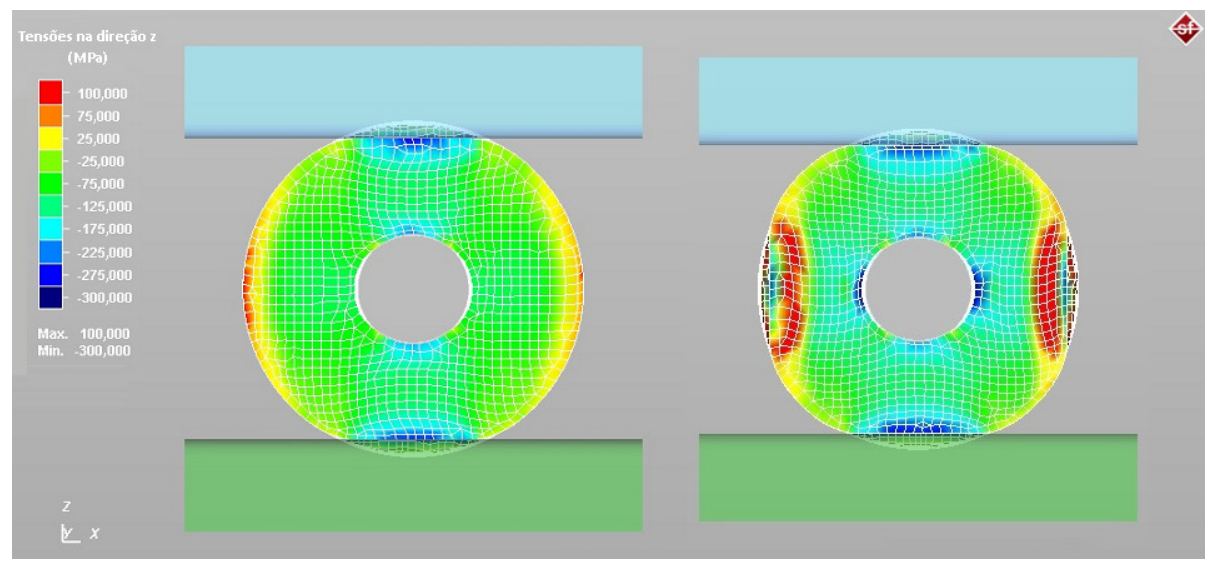

Figura 6. Distribuição das tensões Distribuição de tensões na direção Z para o primeiro recalque.
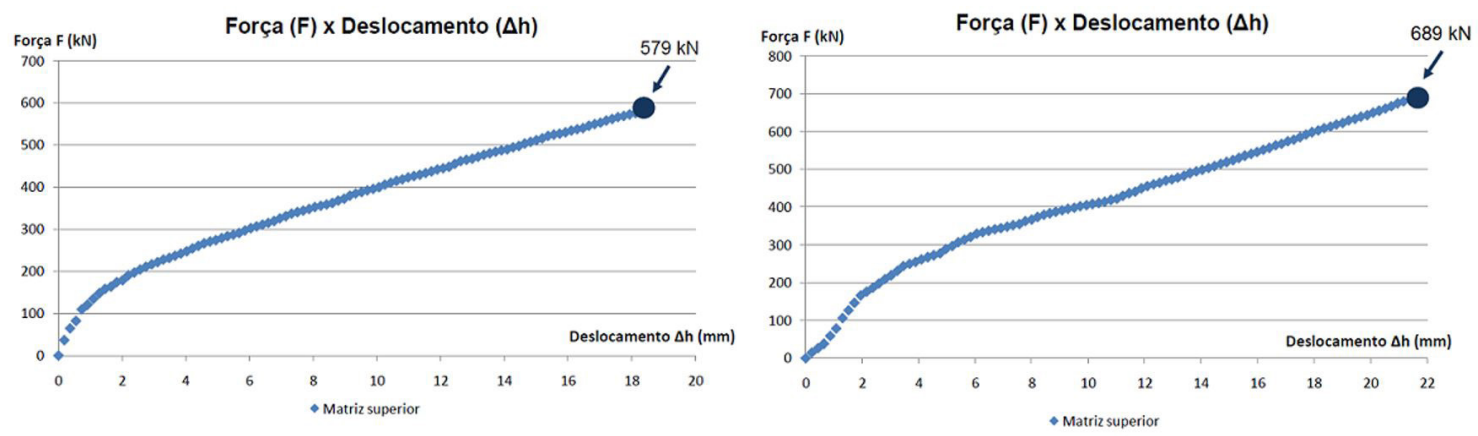

Figura 7. Valores de forças compressivas durante a simulação do forjamento (a) para o primeiro e (b) para o segundo recalque através do software Simufact.
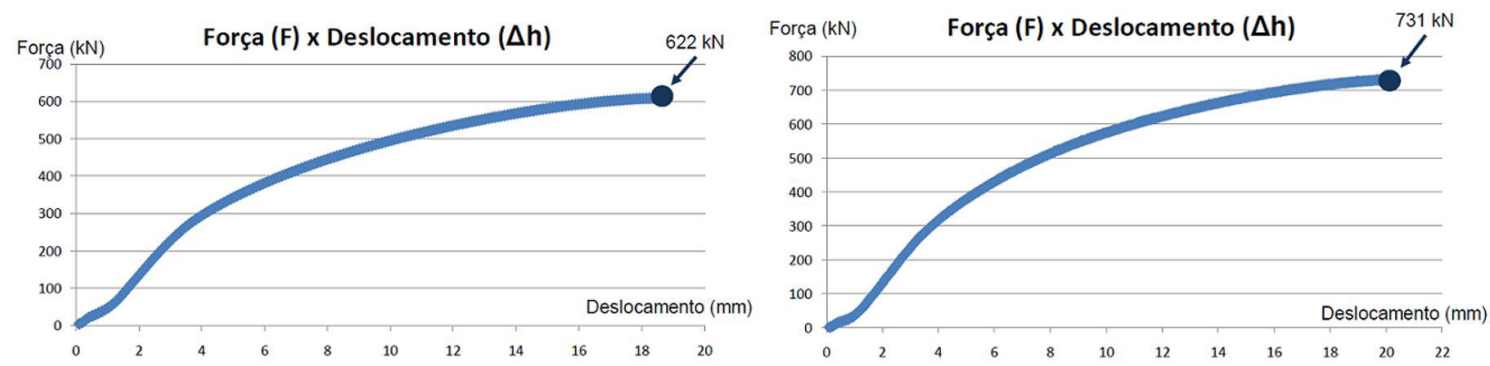

Figura 8. Valores de forças compressivas durante o experimento físico para o primeiro e para o segundo recalque. 
Tabela 2. Forças resultantes da simulação numérica e do experimento físico

\begin{tabular}{cccc}
\hline Compressão & Força (FEM) & $\begin{array}{c}\text { Força } \\
\text { (experimental) }\end{array}$ & $\begin{array}{c}\text { Diferença } \\
\text { Percentual }\end{array}$ \\
\hline $\begin{array}{c}\text { Primeiro } \\
\text { recalque }\end{array}$ & $579 \mathrm{kN}$ & $622 \mathrm{kN}$ & $6,9 \%$ \\
$\begin{array}{c}\text { Segundo } \\
\text { recalque }\end{array}$ & $689 \mathrm{kN}$ & $731 \mathrm{kN}$ & $5,8 \%$ \\
\hline
\end{tabular}

Os valores encontrados a partir da simulação numérica ficaram relativamente próximos aos valores encontrados no experimento físico (Tabela 2), validando o uso da simulação computacional como alternativa aos experimentos físicos.

Há um erro entre $5 \%$ e $10 \%$ em função de diversos fatores do processo, como propriedades metalúrgicas dos materiais, temperatura da peça e das matrizes, coeficiente de atrito entre a peça de trabalho e as matrizes, composição química do material trabalhado e das matrizes, perda de calor durante a condução da peça do forno à prensa hidráulica, aquisição dos dados experimentais durante o forjamento, tensões de escoamento, dentre outros fatores que influenciam nos resultados.

\section{CONCLUSÃO}

Notou-se que as regiões que apresentaram maiores concentrações de deformações equivalentes coincidiram com as regiões que apresentaram maiores concentrações de tensões na direção z pelo software de simulação numérica, onde há valores de maiores intensidades na região central e de menores intensidades nas regiões periféricas, constatando a tendência esperada.

Comparando os valores das forças obtidas através do software de simulação com os valores experimentais, percebeu-se que ficaram próximos entre 5 a $10 \%$. Isso indica que as forças resultantes da simulação numérica são confiáveis, possibilitando fazer comparações com variações e alterações nos métodos de forjamento.

\section{Agradecimentos}

Os autores agradecem o Laboratório de Transformação Mecânica (LdTM), a Universidade Federal do Rio Grande do Sul (UFRGS) e as instituições de apoio financeiro como CNPq e CAPES.

\section{REFERÊNCIAS}

I Irisarri AM, Pelayo A. Failure analysis of an open die forging drop hammer. Engineering Failure Analysis. 2009; 16:1727-1733.

2 Kchaou M, Elleuch R, Desplanques Y, Boidin X, Degallaix G. Failure mechanisms of HI 3 die on relation to the forging process: a case study of brass gas valves. Engineering Failure Analysis. 2010;17(2):403-4I5.

3 Cetlin PR, Helman H. Fundamento da conformação: mecânica dos metais. 2. ed. Belo Horizonte: Artliber; 2005.

4 Choi SK, Chun MS, Van Tyne CJ, Moon YH. Optimization of open die forging of round shapes using FEM analysis. Journal of Materials Processing Technology. 2006; 172:88-95.

5 Ghaei A, Movahhedy MR. Die design for the radial forging process using 3D FEM. Journal of Materials Processing Technology. 2007; 182:534-539.

6 Frota RTC Jr. Avaliação de parâmetros do processo de forjamento a quente em matriz aberta pelo método de elementos finitos [dissertação de mestrado]. São José dos Campos: Instituto Tecnológico da Aeronáutica; 2009.

7 International ASM. ASM Handbook: properties and selection: Irons, steels and high-performance alloys (06I8I). I0. ed. Canadian: ASM International; 1990. 1063 p. vol. I.

8 Cleary PW, Prakash M, Das R, Ha J. Modelling of metal forging using SPH. Applied Mathematical Modelling. 2012;36(8):3836-3855.

$9 \mathrm{Lu} \mathrm{B}, \mathrm{Ou} \mathrm{H}$, Armstrong CG, Rennie A. 3D die shape optimisation for net-shape forging of aerofoil blades. Materials \& Design. 2009;30(7):2490-2500.

10 Kreith, F; Bohn, M. S. Princípios de transferência de calor. São Paulo: Pioneira Thomson Learning; 2003.

I I Börder C. Análise do coeficiente de atrito no ensaio do anel para o forjamento a quente [dissertação de mestrado]. São Paulo: Escola Politécnica, Universidade de São Paulo; 2005.

Recebido em: 23 Set. 2016

Aceito em: 3 Jan. 2017 